\title{
PYTHAGOREAN RATIOS IN ARITHMETIC PROGRESSION, PART II. FOUR PYTHAGOREAN RATIOS
}

\author{
by JEAN LAGRANGE and JOHN LEECH†
}

(Received 23 June, 1992)

1. Introduction. As in [3] let $\{a, b\}$ designate the Pythagorean ratio $\left(a^{2}-b^{2}\right) / 2 a b$ between the sides of a rational right angled triangle. The principal result of [3] is that $\{a, b\}$ is the arithmetic mean of two Pythagorean ratios, and hence is the middle term of a three term arithmetic progression, if and only if $a / b$ is the geometric mean of two Pythagorean ratios. Here in Part II we study sets of four Pythagorean ratios in arithmetic progression. We show that sets of four in consecutive places in an arithmetic progression are closely related to sets of four in the first, second, third and fifth places in a progression; any one of the former sets determines two of the latter sets, and either one of the latter sets determines the other and the former. We construct an infinite sequence of sets of four ratios in consecutive places of arithmetic progressions, the last term of each set being the first term of the next set. These sets are related to solutions of the Diophantine equations $r^{2}=5 p^{2} q^{2} \pm 4\left(p^{4}-2 q^{4}\right)$. Computer searches, in addition to exhibiting enough members of this sequence to enable us to identify it, also exhibited two sets which do not belong to this sequence.

As in [3], $\{a, b\}$ will sometimes be written as $\left\{\begin{array}{l}a \\ b\end{array}\right\}$ in places were $a$ and $b$ are replaced by more elaborate expressions.

2. An example. If we are to have four Pythagorean ratios in arithmetic progression, it is evident that, when these are expressed with a common denominator, this will be a highly composite number. So we can choose a suitably composite number and list the Pythagorean ratios which can share it as denominator. In this way we find, for the denominator 420 , that the possible numerators include the arithmetic progression 175 , $513,851,1189$, corresponding to the Pythagorean ratios $\{3,2\},\{14,5\},\{30,7\},\{35,6\}$, which is the simplest example. (This is similar to the search showing that the denominator 120 gives the simplest example of two Pythagorean ratios, $\{15,4\}$ and $\{10,3\}$, whose sum $\{20,3\}$ and difference $\{5,4\}$ are also Pythagorean ratios [2].) Computer searches finding other examples are described in later Sections.

This arithmetic progression of four Pythagorean ratios includes two progressions of three ratios, each of which, as found in [3], is one of a set of four such triads. These may be set out as arrays thus, where three ratios in any row or column are in arithmetic progression.

$\begin{array}{cccccc}\{3,2\} & \{14,5\} & \{30,7\} & \{14,5\} & \{30,7\} & \{35,6\} \\ \{8,3\} & & \{3,8\} & \{40,7\} & & \{7,40\} \\ \{4,1\} & \{5,14\} & \{4,35\} & \{35,4\} & \{7,30\} & \{5,84\}\end{array}$

In addition to the shared members in the top rows, forming the arithmetic progression of four terms, there is also a shared member $\{35,4\}$, appearing with opposite signs in the 
two arrays. As a consequence of this, we find two arithmetic progressions of five terms of which four are Pythagorean ratios, namely $\{35,4\},\{40,7\},\{14,5\}, *,\{1,4\}$ and $\{4,35\}$, $\{3,8\},\{30,7\}, *,\{84,5\}$. We shall see in the next Section that the corresponding two progressions are determined by any arithmetic progression of four Pythagorean ratios in consecutive places.

3. Arithmetic progressions of five terms including four Pythagorean ratios. Any arithmetic progression of four Pythagorean ratios $\left\{a_{i}, b_{i}\right\}$ in consecutive places includes two triads, from which we can form arrays of four triads of Pythagorean ratios (Part I, Section 8), namely:

$$
\begin{aligned}
& \left\{a_{1}, b_{1}\right\} \quad\left\{a_{2}, b_{2}\right\} \quad\left\{a_{3}, b_{3}\right\} \\
& \left\{\begin{array}{l}
a_{1} a_{3}+b_{1} b_{3} \\
b_{1} a_{3}-a_{1} b_{3}
\end{array}\right\} \quad\left\{\begin{array}{l}
a_{1} a_{3}+b_{1} b_{3} \\
a_{1} b_{3}-b_{1} a_{3}
\end{array}\right\} \\
& \left\{\begin{array}{l}
a_{1} a_{2}+b_{1} b_{2} \\
b_{1} a_{2}-a_{1} b_{2}
\end{array}\right\} \quad\left\{b_{2}, a_{2}\right\} \quad\left\{\begin{array}{l}
a_{2} a_{3}+b_{2} b_{3} \\
a_{2} b_{3}-b_{2} a_{3}
\end{array}\right\} \\
& \left\{a_{2}, b_{2}\right\} \quad\left\{a_{3}, b_{3}\right\} \quad\left\{a_{4}, b_{4}\right\} \\
& \left\{\begin{array}{l}
a_{2} a_{4}+b_{2} b_{4} \\
b_{2} a_{4}-a_{2} b_{4}
\end{array}\right\} \quad\left\{\begin{array}{l}
a_{2} a_{4}+b_{2} b_{4} \\
a_{2} b_{4}-b_{2} a_{4}
\end{array}\right\} \\
& \left\{\begin{array}{l}
a_{2} a_{3}+b_{2} b_{3} \\
b_{2} a_{3}-a_{2} b_{3}
\end{array}\right\} \quad\left\{b_{3}, a_{3}\right\} \quad\left\{\begin{array}{l}
a_{3} a_{4}+b_{3} b_{4} \\
a_{3} b_{4}-b_{3} a_{4}
\end{array}\right\}
\end{aligned}
$$

We see that the same element $\left\{a_{2} a_{3}+b_{2} b_{3}, a_{2} b_{3}-b_{2} a_{3}\right\}$ appears, with opposite signs, in a lower corner of each of these arrays. Thus the column containing this element in each array can be used to interpolate an arithmetic mean between two terms of the triad on the bottom row of the other array. We therefore have the following two arithmetic progressions of five terms of which the first, second, third and fifth are Pythagorean ratios:

$$
\begin{aligned}
& \left\{\begin{array}{l}
a_{2} a_{3}+b_{2} b_{3} \\
a_{2} b_{3}-b_{2} a_{3}
\end{array}\right\} \quad\left\{\begin{array}{l}
a_{1} a_{3}+b_{1} b_{3} \\
a_{1} b_{3}-b_{1} a_{3}
\end{array}\right\} \quad\left\{a_{3}, b_{3}\right\} \quad *\left\{\begin{array}{l}
a_{3} a_{4}+b_{3} b_{4} \\
b_{3} a_{4}-a_{3} b_{4}
\end{array}\right\} \\
& \left\{\begin{array}{l}
a_{2} a_{3}+b_{2} b_{3} \\
b_{2} a_{3}-a_{2} b_{3}
\end{array}\right\} \quad\left\{\begin{array}{l}
a_{2} a_{4}+b_{2} b_{4} \\
b_{2} a_{4}-a_{2} b_{4}
\end{array}\right\} \quad\left\{a_{2}, b_{2}\right\} \quad * \quad\left\{\begin{array}{l}
a_{1} a_{2}+b_{1} b_{2} \\
a_{1} b_{2}+b_{1} a_{2}
\end{array}\right\}
\end{aligned}
$$

Conversely, we can see similarly that this whole configuration is determined by the elements $\left\{\alpha_{1}, \beta_{1}\right\},\left\{\alpha_{2}, \beta_{2}\right\},\left\{\alpha_{2}, \beta_{3}\right\}, *,\left\{\alpha_{5}, \beta_{5}\right\}$ in a progression of five terms. This progression also includes two triads, which also determine arrays of four triads with a further common member:

$$
\begin{array}{ccc}
\left\{\alpha_{1}, \beta_{1}\right\} & \left\{\alpha_{2}, \beta_{2}\right\} & \left\{\alpha_{3}, \beta_{3}\right\} \\
\left\{\begin{array}{l}
\alpha_{1} \alpha_{3}+\beta_{1} \beta_{3} \\
\beta_{1} \alpha_{3}-\alpha_{1} \beta_{3}
\end{array}\right\} & \left\{\begin{array}{l}
\alpha_{1} \alpha_{3}+\beta_{1} \beta_{3} \\
\alpha_{1} \beta_{3}-\beta_{1} \alpha_{3}
\end{array}\right\} \\
\left\{\begin{array}{l}
\alpha_{1} \alpha_{2}+\beta_{1} \beta_{2} \\
\beta_{1} \alpha_{2}-\alpha_{1} \beta_{2}
\end{array}\right\} & \left\{\beta_{2}, \alpha_{2}\right\} & \left\{\begin{array}{l}
\alpha_{2} \alpha_{3}+\beta_{2} \beta_{3} \\
\alpha_{2} \beta_{3}-\beta_{2} \alpha_{3}
\end{array}\right\}
\end{array}
$$




$$
\begin{array}{ccc}
\left\{\alpha_{1}, \beta_{1}\right\} & \left\{\alpha_{3}, \beta_{3}\right\} & \left\{\alpha_{5}, \beta_{5}\right\} \\
\left\{\begin{array}{l}
\alpha_{1} \alpha_{5}+\beta_{1} \beta_{5} \\
\beta_{1} \alpha_{5}-\alpha_{1} \beta_{5}
\end{array}\right\} & \left\{\begin{array}{l}
\alpha_{1} \alpha_{5}+\beta_{1} \beta_{5} \\
\alpha_{1} \beta_{5}-\beta_{1} \alpha_{5}
\end{array}\right\} \\
\left\{\begin{array}{l}
\alpha_{1} \alpha_{3}+\beta_{1} \beta_{3} \\
\beta_{1} \alpha_{3}-\alpha_{1} \beta_{3}
\end{array}\right\} & \left\{\beta_{3}, \alpha_{3}\right\} & \left\{\begin{array}{l}
\alpha_{3} \alpha_{5}+\beta_{3} \beta_{5} \\
\alpha_{3} \beta_{5}-\beta_{3} \alpha_{5}
\end{array}\right\}
\end{array}
$$

We see that these arrays are equivalent to the two determined by the four Pythagorean ratios in consecutive places in an arithmetic progression, and we can similarly pick out from them the progression

$$
\left\{\begin{array}{l}
\alpha_{2} \alpha_{3}+\beta_{2} \beta_{3} \\
\alpha_{2} \beta_{3}-\beta_{2} \alpha_{3}
\end{array}\right\} \quad\left\{\begin{array}{l}
\alpha_{1} \alpha_{3}+\beta_{1} \beta_{3} \\
\alpha_{1} \beta_{3}-\beta_{1} \alpha_{3}
\end{array}\right\} \quad\left\{\alpha_{3}, \beta_{3}\right\} \quad\left\{\begin{array}{l}
\alpha_{3} \alpha_{5}+\beta_{3} \beta_{5} \\
\beta_{3} \alpha_{5}-\alpha_{3} \beta_{5}
\end{array}\right\}
$$

of consecutive terms, and the other progression

$$
\left\{\alpha_{1}, \beta_{1}\right\} \quad\left\{\begin{array}{l}
\alpha_{1} \alpha_{5}+\beta_{1} \beta_{5} \\
\beta_{1} \alpha_{5}-\alpha_{1} \beta_{5}
\end{array}\right\} \quad\left\{\begin{array}{l}
\alpha_{1} \alpha_{3}+\beta_{1} \beta_{3} \\
\beta_{1} \alpha_{3}-\alpha_{1} \beta_{3}
\end{array}\right\} \quad * \quad\left\{\begin{array}{l}
\alpha_{1} \alpha_{2}+\beta_{1} \beta_{2} \\
\beta_{1} \alpha_{2}-\alpha_{1} \beta_{2}
\end{array}\right\}
$$

of five terms including four Pythagorean ratios.

4. Searches for solutions. The initial search by hand examined as denominators numbers, such as 120 and 840 , which are products of a power of 2 by other small primes. This exhibited the example of Section 2.

In view of the relationship (Part I) between arithmetic progressions of three Pythagorean ratios and pairs of Pythagorean ratios whose products and quotients are squares, we took examples of the latter from an extensive tabulation [1] of solutions of

$$
\left\{\alpha_{i-1}, \beta_{i-1}\right\}\left\{\alpha_{i+1}, \beta_{i+1}\right\}=\frac{\alpha_{i}^{2}+\beta_{i}^{2}}{2 \alpha_{i} \beta_{i}} .
$$

Examples for which both $\alpha_{i}^{2} \alpha_{i+1}^{2}+\beta_{i}^{2} \beta_{i+1}^{2}$ and $\alpha_{i}^{2} \beta_{i+1}^{2}+\beta_{i}^{2} \alpha_{i+1}^{2}$ are squares had been identified in this tabulation. From these, we calculated the corresponding arrays of triads of Pythagorean ratios in arithmetic progression, and checked their entries for overlap. This search produced two examples to add to that of Section 2, so we had the examples

$$
\begin{array}{cccc}
\{3,2\} & \{14,5\} & \{30,7\} & \{35,6\}, \\
\{35,6\} & \{48,11\} & \{165,56\} & \{176,105\}, \\
\{728,51\} & \{85,11\} & \{440,273\} & \{2341,420\} .
\end{array}
$$

The repetition of $\{35,6\}$ was noted, but at that stage we could not regard it as being more than coincidence.

A further search used a method based on the analysis of the next Section, and this produced two further examples:

$$
\begin{array}{cccc}
\{1395,476\} & \{4104,2635\} & \{459,665\} & \{1736,4845\}, \\
\{176,105\} & \{30,259\} & \{112,2035\} & \{111,3080\} .
\end{array}
$$

The repetition of $\{176,105\}$ was noted, and this led us to the discovery of an infinite sequence of tetrads of Pythagorean ratios in arithmetic progression, the last member of each tetrad being the first member of the next tetrad. 
5. An equivalent system of Diophantine equations. If we express four Pythagorean ratios in arithmetic progression with a common denominator, their numerators are in arithmetic progression, and we have a solution of a system of Diophantine equations which may be written as

$$
\begin{aligned}
a^{2}+\left(b-3 m^{2} c\right)^{2} & =m^{2}(f-g)^{2}, \\
a^{2}+\left(b-m^{2} c\right)^{2} & =m^{2}(d-e)^{2}, \\
a^{2}+\left(b+m^{2} c\right)^{2} & =m^{2}(d+e)^{2}, \\
a^{2}+\left(b+3 m^{2} c\right)^{2} & =m^{2}(f+g)^{2} .
\end{aligned}
$$

It is easily seen that if the right hand squares have a common factor $m^{2}$, this has to appear on the left hand sides in the position shown. We do not exclude $m=1$, but we have found no example of it.

This system is equivalent to

$$
\begin{gathered}
a^{2}+b^{2}+c^{2} m^{4}=m^{2}\left(d^{2}+e^{2}\right), \\
a^{2}+b^{2}+9 c^{2} m^{4}=m^{2}\left(f^{2}+g^{2}\right), \\
b c=d e, \quad 3 b c=f g .
\end{gathered}
$$

The first two of these may be replaced by

$$
\begin{aligned}
8 c^{2} m^{2} & =f^{2}+g^{2}-d^{2}-e^{2}, \\
8\left(a^{2}+b^{2}\right) & =m^{2}\left(9\left(d^{2}+e^{2}\right)-\left(f^{2}+g^{2}\right)\right) .
\end{aligned}
$$

To satisfy the other two, we set

$$
d=\alpha \delta, \quad e=\beta \gamma, \quad f=\alpha \gamma, \quad g=3 \beta \delta,
$$

from which we obtain

and

$$
\begin{aligned}
b c & =\alpha \beta \gamma \delta \\
8 c^{2} m^{2} & =\alpha^{2}\left(\gamma^{2}-\delta^{2}\right)-\beta^{2}\left(\gamma^{2}-9 \delta^{2}\right),
\end{aligned}
$$

which reduces to

$$
\begin{aligned}
(8 a c)^{2} & =64\left(a^{2}+b^{2}\right) c^{2}-64 b^{2} c^{2} \\
& =8 c^{2} m^{2}\left(9\left(d^{2}+e^{2}\right)-\left(f^{2}+g^{2}\right)\right)-64 b^{2} c^{2},
\end{aligned}
$$

$$
(8 a c)^{2}=-\left(\alpha^{2}-\beta^{2}\right)\left(\alpha^{2}-9 \beta^{2}\right)\left(\gamma^{2}-\delta^{2}\right)\left(\gamma^{2}-9 \delta^{2}\right)
$$

on substituting for $d, e, f, g, b c$ and $8 c^{2} m^{2}$.

To search for solutions of this system, we seek integers such that

and

$$
-\left(\alpha^{2}-\beta^{2}\right)\left(\alpha^{2}-9 \beta^{2}\right)\left(\gamma^{2}-\delta^{2}\right)\left(\gamma^{2}-9 \delta^{2}\right)
$$

$$
2\left(\alpha^{2}\left(\gamma^{2}-\delta^{2}\right)-\beta^{2}\left(\gamma^{2}-9 \delta^{2}\right)\right)
$$

are both squares. We can assume $(\alpha, \beta)=(\gamma, \delta)=1$. Our computer search for square values of

$$
-\left(\alpha^{2}-\beta^{2}\right)\left(\alpha^{2}-9 \beta^{2}\right)\left(\gamma^{2}-\delta^{2}\right)\left(\gamma^{2}-9 \delta^{2}\right)
$$


is based on listing, for a suitable range of $\alpha, \beta$, the values of $\left(\alpha^{2}-\beta^{2}\right)\left(\alpha^{2}-9 \beta^{2}\right)=k l^{2}$, where $k$ is square free. Two tables of $|k|$ are kept, according to the sign of $k$, and the corresponding values of $\alpha, \beta$ are listed with $k$. Whenever entries occur for the same value of $k$ in both tables, we check whether $\alpha^{2}\left(\gamma^{2}-\delta^{2}\right)-\beta^{2}\left(\gamma^{2}-9 \delta^{2}\right)$ is twice a square. We took $\alpha, \beta<2000$ and retained only $|k|<10^{6}$.

We found the following five solutions:

\begin{tabular}{rrrr}
$\alpha$ & $\beta$ & \multicolumn{1}{c}{$\gamma$} & \multicolumn{1}{c}{$\delta$} \\
1 & 2 & 31 & 11 \\
19 & 3 & 83 & 79 \\
59 & 71 & 79 & 53 \\
75 & 37 & 11 & 389 \\
35 & 13 & 1151 & 1217
\end{tabular}

corresponding to the five tetrads of Pythagorean ratios in arithmetic progression listed in Section 4.

6. An infinite family of solutions. It has been noted that three of the five tetrads of Pythagorean ratios found are linked by having shared end members. They have other features distinguishing them from the other two, such as that $a^{2}+b^{2}$, which is always divisible by $m^{2}$, is divisible by $\mathrm{m}^{3}$ in these three solutions but not in the other two. We therefore looked for a family relationship between them. The rest of this paper is devoted to the study of this family.

Each of the solutions listed in Section 5 appeared twice in the search, since if $\alpha, \beta, \gamma$, $\delta$ is a solution, then so is $3 \beta, \alpha, 3 \delta, \gamma$, except for the last solution, whose second appearance was not within our range of search. It was due to reappear as

$$
39, \quad 35=7 \cdot 5, \quad 3651=7^{4}+2 \cdot 5^{4}, \quad 1151=7^{4}-2 \cdot 5^{4} .
$$

The first two solutions can be expressed similarly as

$$
\begin{array}{llll}
12, & 2, & 33=2 \cdot 2^{4}+1, & 31=2 \cdot 2^{4}-1, \\
19, & 3, & 83=3^{4}+2, & 79=3^{4}-2 .
\end{array}
$$

We therefore set out to generalize these by setting $\gamma=p^{4}+2 q^{4}, \delta=p^{4}-2 q^{4}, \beta=p q$. We now have

giving

$$
\begin{gathered}
\gamma^{2}-\delta^{2}=8 p^{4} q^{4} \\
\gamma^{2}-9 \delta^{2}=-8\left(p^{4}-q^{4}\right)\left(p^{4}-4 q^{4}\right),
\end{gathered}
$$

$$
\begin{gathered}
b c=\alpha \beta\left(p^{4}+2 q^{4}\right)\left(p^{4}-2 q^{4}\right), \\
c^{2} m^{2}=\alpha^{2} p^{4} q^{4}+\beta^{2}\left(p^{4}-q^{4}\right)\left(p^{4}-4 q^{4}\right) \\
a^{2} c^{2}=\left(\alpha^{2}-\beta^{2}\right)\left(\alpha^{2}-9 \beta^{2}\right) p^{4} q^{4}\left(p^{4}-q^{4}\right)\left(p^{4}-4 q^{4}\right) .
\end{gathered}
$$

With $\beta=p q$ and setting $c=\beta$, we obtain

$$
\begin{gathered}
b=\alpha\left(p^{4}+2 q^{4}\right)\left(p^{4}-2 q^{4}\right), \\
m^{2}=\alpha^{2} p^{2} q^{2}+\left(p^{4}-q^{4}\right)\left(p^{4}-4 q^{4}\right), \\
a^{2}=\left(\alpha^{2}-p^{2} q^{2}\right)\left(\alpha^{2}-9 p^{2} q^{2}\right) p^{2} q^{2}\left(p^{4}-q^{4}\right)\left(p^{4}-4 q^{4}\right) .
\end{gathered}
$$


To satisfy this last equation, we put

$$
\alpha^{2}=5 p^{2} q^{2}+4 \varepsilon\left(p^{4}-2 q^{4}\right), \quad \text { with } \quad \varepsilon= \pm 1,
$$

giving

$$
\begin{aligned}
\alpha^{2}-p^{2} q^{2} & =4\left(\varepsilon p^{2}-q^{2}\right)\left(p^{2}+2 \varepsilon q^{2}\right) \\
\alpha^{2}-9 p^{2} q^{2} & =4\left(\varepsilon p^{2}+q^{2}\right)\left(p^{2}-2 \varepsilon q^{2}\right)
\end{aligned}
$$

We thus have

$$
a=4 p q\left(p^{4}-q^{4}\right)\left(p^{4}-4 q^{4}\right)
$$

and

$$
\begin{aligned}
m^{2} & =p^{8}+4 q^{8}+4 \varepsilon p^{2} q^{2}\left(p^{4}-2 q^{4}\right) \\
& =\left(2 p^{2} q^{2}+\varepsilon\left(p^{4}-2 q^{4}\right)\right)^{2},
\end{aligned}
$$

so

$$
m=2 p^{2} q^{2}+\varepsilon\left(p^{4}-2 q^{4}\right) .
$$

Thus from any solution of

$$
r^{2}=5 p^{2} q^{2}+4 \varepsilon\left(p^{4}-2 q^{4}\right)
$$

we obtain a solution of

$$
\begin{aligned}
a^{2}+\left(b \pm c m^{2}\right)^{2} & =m^{2}(d \pm e)^{2}, \\
a^{2}+\left(b \pm 3 c m^{2}\right)^{2} & =m^{2}(f \pm g)^{2},
\end{aligned}
$$

with

$$
\begin{aligned}
a & =4 p q\left(p^{4}-q^{4}\right)\left(p^{4}-4 q^{4}\right), \\
b & =r\left(p^{4}+2 q^{4}\right)\left(p^{4}-2 q^{4}\right), \\
c & =p q \\
m & =2 p^{2} q^{2}+\varepsilon\left(p^{4}-2 q^{4}\right), \\
d & =r\left(p^{4}-2 q^{4}\right), \\
e & =p q\left(p^{4}+2 q^{4}\right), \\
f & =r\left(p^{4}+2 q^{4}\right), \\
g & =3 p q\left(p^{4}-2 q^{4}\right) .
\end{aligned}
$$

7. The Diophantine equations $r^{2}=5 p^{2} q^{2} \pm 4\left(p^{4}-2 q^{4}\right)$. Our attention is thus drawn to the study of integer solutions of the two equations

$$
\begin{aligned}
& r^{2}=5 p^{2} q^{2}+4\left(p^{4}-2 q^{4}\right), \\
& r^{2}=5 p^{2} q^{2}-4\left(p^{4}-2 q^{4}\right) .
\end{aligned}
$$

For the purpose of this study we set $x=p / q, y=r / q^{2}$, and examine the rational points on the plane quartic curves

$$
\begin{array}{ll}
\Gamma_{r}: & y^{2}=4 x^{4}+5 x^{2}-8 \\
\Gamma_{i}: & y^{\prime 2}=-4 x^{\prime 4}+5 x^{\prime 2}+8
\end{array}
$$


We can pass from $\Gamma_{r}$ to $\Gamma_{i}$ by setting $x=i x^{\prime}, y=i y^{\prime}$. We may thus consider the curve

$$
\Gamma: \quad Y^{2}=5 X^{4}+5 X^{2}-8
$$

over $\mathbb{Q}(i) . \Gamma_{r}$ is the real part of $\Gamma ; \Gamma_{i}$ is associated with the imaginary part of $\Gamma$ (those points whose coordinates are pure imaginary), denoted $i \Gamma_{r}$.

The study of this curve is standard. From two rational points (over $\mathbb{Q}(i)$ ) we deduce a third from the intersection with $\Gamma$ of the parabola $Y=2 X^{2}+\lambda X+\mu$ passing through the first two points. We thus obtain

$$
\begin{gathered}
X_{3}=\frac{Y_{1} X_{2}-X_{1} Y_{2}}{2\left(X_{1}^{2}-X_{2}^{2}\right)}=\frac{2\left(X_{1} X_{2}+2\right)}{Y_{1} X_{2}+X_{1} Y_{2}} \\
Y_{3}=-X_{3} \frac{X_{1} Y_{1}-X_{2} Y_{2}}{X_{1}^{2}-X_{2}^{2}}+2 X_{1} X_{2}
\end{gathered}
$$

We have to change the sign of $X_{3}$ to obtain the addition formula for the Abelian group of rational points of $\Gamma$ with the point at infinity as the zero element.

By identifying the two points on the curve and using the tangent parabola, we obtain the duplication formula

$$
X_{2}=\frac{2+X_{1}^{4}}{X_{1} Y_{1}}, \quad Y_{2}=2 X_{2}^{2}+\frac{2-X_{1}^{4}}{X_{1}^{2}}
$$

We can assing a "parity" to points on $\Gamma$, so that points on $\Gamma_{r}$ are "even" and points on $i \Gamma_{r}$ are "odd"; the sum of two odd points or two even points is even, while the sum of an odd point and an even point is odd.

The point $(i, 3 i)$ of $i \Gamma_{r}$ is not a torsion point, and it generates an infinite sequence of points which are alternatively on $i \Gamma_{r}$ and $\Gamma_{r}$. Thus we obtain a sequence of points $\left(x_{n}, y_{n}\right)$ alternately on $\Gamma_{r}$ and $\Gamma_{i}$. It seemed likely, and Professor R. K. Guy (private communication) has kindly proved for us, that the curves $\Gamma_{r}$ and $\Gamma_{i}$ are of rank 1 , so this generates all the rational points on these curves. (We add parenthetically that the rank of $\Gamma$ over $\mathbb{Q}(i)$ is at least 2 , since the point $(2 i, 6)$ is an independent non-torsion point, but it does not generate points on $\Gamma_{r}$ or $\Gamma_{i}$.)

8. Recurrence relations. The foregoing analysis has shown the existence of an infinite sequence of solutions of the equations $r^{2}=5 p^{2} q^{2} \pm 4\left(p^{4}-2 q^{4}\right)$. Putting $\varepsilon_{n}=(-1)^{n}$ and $n_{n}=2-\varepsilon_{n}$ (so $n_{2 m}=1, n_{2 m+1}=3$ ), we can obtain the following recurrence relations for solutions of

$$
r_{n}^{2}=5 p_{n}^{2} q_{n}^{2}+4 \varepsilon_{n}\left(p_{n}^{4}-2 q_{n}^{4}\right)
$$

by adding or subtracting the points $(i, 3 i)$ and $(-1,1)$, where $(-1,1)=(i, 3 i)+(i, 3 i)$ :

$$
\begin{aligned}
\eta_{n} p_{n-1} p_{n+1} & =p_{n}^{2}-2 \varepsilon_{n} q_{n}^{2}, & \eta_{n} q_{n-1} q_{n+1} & =q_{n}^{2}+\varepsilon_{n} p_{n}^{2}, \\
p_{n-2} p_{n+2} & =p_{n}^{2}+2 \varepsilon_{n} q_{n}^{2}, & q_{n-2} q_{n+2} & =q_{n}^{2}-\varepsilon_{n} p_{n}^{2}, \\
2 \eta_{n} p_{n-1} q_{n+1} & =r_{n}+3 p_{n} q_{n}, & 2 \eta_{n} q_{n-1} p_{n+1} & =r_{n}-3 p_{n} q_{n}, \\
2 p_{n-2} q_{n+2} & =p_{n} q_{n}+r_{n}, & 2 q_{n-2} p_{n+2} & =p_{n} q_{n}-r_{n},
\end{aligned}
$$


with initial values $q_{0}=0, p_{0}=p_{1}=p_{2}=q_{1}=q_{2}=1$. (The recurrence relations involving only subscripts $n, n \pm 2$ allow us to generate solutions of either one of the equations $r^{2}=5 p^{2} q^{2} \pm 4\left(p^{4}-2 q^{4}\right)$ separately, should these be needed.) The first few solutions are listed below.

\begin{tabular}{rrrr}
$n$ & \multicolumn{1}{c}{$p_{n}$} & $q_{n}$ & \multicolumn{1}{c}{$r_{n}$} \\
0 & 1 & 0 & 2 \\
1 & 1 & 1 & 3 \\
2 & 1 & 1 & 1 \\
3 & -1 & 2 & 12 \\
4 & 3 & 1 & -19 \\
5 & -7 & 5 & -39 \\
6 & 11 & -8 & -254 \\
7 & 1 & 37 & -3873 \\
8 & 83 & -57 & 14741 \\
9 & 391 & 274 & -94812 \\
10 & 1217 & 455 & 3156697
\end{tabular}

It will be seen that the recurrence relations attach signs to the numbers involved. Although these are not relevant to the equations $r_{n}^{2}=5 p_{n}^{2} q_{n}^{2} \pm 4\left(p_{n}^{4}-2 q_{n}^{4}\right)$, in which only even powers appear, they are necessary for some of the formulae in the next Section.

9. Numerical solutions in the infinite family. We can now retrace our steps and exhibit members of the infinite family of tetrads of Pythagorean ratios in arithmetic progression. We have

$$
\begin{aligned}
a_{n} & =4 p_{n} q_{n}\left(p_{n}^{4}-q_{n}^{4}\right)\left(p_{n}^{4}-4 q_{n}^{4}\right) / \eta_{n}^{2} \\
b_{n} & =r_{n}\left(p_{n}^{4}+2 q_{n}^{4}\right)\left(p_{n}^{4}-2 q_{n}^{4}\right) / \eta_{n}^{2}, \\
c_{n} & =p_{n} q_{n} \\
m_{n} & =\left(2 p_{n}^{2} q_{n}^{2}+\varepsilon_{n}\left(p_{n}^{4}-2 q_{n}^{4}\right)\right) / \eta_{n} \\
d_{n} & =r_{n}\left(p_{n}^{4}-2 q_{n}^{4}\right) / \eta_{n} \\
e_{n} & =p_{n} q_{n}\left(p_{n}^{4}+2 q_{n}^{4}\right) / \eta_{n} \\
f_{n} & =r_{n}\left(p_{n}^{4}+2 q_{n}^{4}\right) / \eta_{n} \\
g_{n} & =3 p_{n} q_{n}\left(p_{n}^{4}-2 q_{n}^{4}\right) / \eta_{n}
\end{aligned}
$$

with $\varepsilon_{n}=(-1)^{n}$ and $\eta_{n}=2-\varepsilon_{n}$, giving solutions of the system

$$
\begin{aligned}
a^{2}+\left(b \pm c m^{2}\right)^{2} & =m^{2}(d \pm e)^{2}, \\
a^{2}+\left(b \pm 3 c m^{2}\right)^{2} & =m^{2}(f \pm g)^{2} .
\end{aligned}
$$

Here the common factor $\eta_{n},=3$ when $n$ is odd, has been taken out. When $n$ is a multiple of $3, q_{n}$ and $r_{n}$ are both even, and we can remove a common factor 2 from $a, b, c, d, e, f$, 
g. The first few non-trivial solutions are:

$\begin{array}{rrrrr}n=3 & 4 & 5 & 6 \\ a & =420 & 73920 & 2735040 & 3234868560 \\ b=682 & 124583 & 18209971 & 18700752159 \\ c=1 & 3 & 35 & 44 \\ m=13 & 97 & 433 & 21937 \\ d=62 & 1501 & 42595 & 819023 \\ e=11 & 249 & 14963 & 1004652 \\ f=66 & 1577 & 47643 & 2899791 \\ g=31 & 711 & 40285 & 851268\end{array}$

The Pythagorean ratios in arithmetic progression are the ratios $\left(b+k c m^{2}\right) / a$ with $k=-3,-1,1,3$. Their generators may be expressed as

$$
\begin{array}{ll}
\left\{\begin{array}{c}
p_{n-2} q_{n-2} p_{n+1} q_{n+1} \\
p_{n-1} q_{n-1} p_{n} q_{n}
\end{array}\right\}, & \left\{\begin{array}{c}
p_{n-2} q_{n-1} p_{n} q_{n+2} \\
q_{n-2} p_{n-1} q_{n} p_{n+2}
\end{array}\right\}, \\
\left\{\begin{array}{c}
q_{n-2} p_{n} q_{n+1} p_{n+2} \\
p_{n-2} q_{n} p_{n+1} q_{n+2}
\end{array}\right\}, & \left\{\begin{array}{c}
p_{n} q_{n} p_{n+1} q_{n+1} \\
p_{n-1} q_{n-1} p_{n+2} q_{n+2}
\end{array}\right\},
\end{array}
$$

It will be seen that the last pair of generators has the same form as the first pair, with the subscripts advanced by 1 and the generators interchanged, corresponding to a change of sign of the ratio. Taking this sign into account, we see that the last term of each progression can be used as the first term of the next progression, as we had found empirically. The first few tetrads are:

$\begin{array}{cccc}\{3,2\} & \{14,5\} & \{30,7\} & \{35,6\}, \\ \{35,6\} & \{48,11\} & \{165,56\} & \{176,105\}, \\ \{176,105\} & \{30,259\} & \{112,2035\} & \{111,3080\}, \\ \{111,3080\} & \{4648,9405\} & \{33781,1368\} & \{165585,3256\}, \\ \{165585,3256\} & \{795865,15344\} & \{5890178,111435\} & \{9427792,175047\} .\end{array}$

Among observations that may be made, we remark that the generators of the shared ratios $\{35,6\},\{176,105\},\{111,3080\},\{165585,3256\}, \ldots$ have the property

$$
\begin{aligned}
35^{2}+6^{2} & =13 \cdot 97, \\
176^{2}+105^{2} & =97 \cdot 433, \\
111^{2}+3080^{2} & =433 \cdot 21937, \\
165585^{2}+3256^{2} & =21937 \cdot 1250353,
\end{aligned}
$$


where the factors on the right are the values of $m$ in the two progressions sharing this ratio (and the initial ratio $\{3,2\}$ has $3^{2}+2^{2}=13$, the value of $m$ for the first progression). This can be proved generally valid for this family of progressions, and it was one of the empirical observations which led us to its discovery.

Each progression of four ratios contains two progressions of three ratios, each of which is one of an array of four such triads. These have the following generators:

$$
\begin{aligned}
& \left\{\begin{array}{c}
p_{n-2} q_{n-2} p_{n+1} q_{n+1} \\
p_{n-1} q_{n-1} p_{n} q_{n}
\end{array}\right\} \quad\left\{\begin{array}{c}
p_{n-2} q_{n-1} p_{n} q_{n+2} \\
q_{n-2} p_{n-1} q_{n} p_{n+2}
\end{array}\right\} \quad\left\{\begin{array}{l}
q_{n-2} p_{n} q_{n+1} p_{n+2} \\
p_{n-2} q_{n} p_{n+1} q_{n+2}
\end{array}\right\} \\
& \left\{\begin{array}{l}
p_{n-2} p_{n}^{2} p_{n+1} \\
2 q_{n-2} q_{n}^{2} q_{n+1}
\end{array}\right\} \\
& \left\{\begin{array}{l}
q_{n-2} q_{n-1} p_{n}^{2} \\
p_{n-2} p_{n-1} q_{n}^{2}
\end{array}\right\} \quad\left\{\begin{array}{l}
q_{n-2} p_{n-1} q_{n} p_{n+2} \\
p_{n-2} q_{n-1} p_{n} q_{n+2}
\end{array}\right\} \quad\left\{\begin{array}{c}
p_{n}^{2} q_{n}^{2} \\
p_{n-2} q_{n-2} p_{n+2} q_{n+2}
\end{array}\right\} \\
& \left\{\begin{array}{l}
p_{n-2} q_{n-1} p_{n} q_{n+2} \\
q_{n-2} p_{n-1} q_{n} p_{n+2}
\end{array}\right\} \quad\left\{\begin{array}{l}
q_{n-2} p_{n} q_{n+1} p_{n+2} \\
p_{n-2} q_{n} p_{n+1} q_{n+2}
\end{array}\right\} \quad\left\{\begin{array}{c}
p_{n} q_{n} p_{n+1} q_{n+1} \\
p_{n-1} q_{n-1} p_{n+2} q_{n+2}
\end{array}\right\} \\
& \left\{\begin{array}{l}
2 q_{n-1} q_{n}^{2} q_{n+2} \\
p_{n-1} p_{n}^{2} p_{n+2}
\end{array}\right\} \quad\left\{\begin{array}{l}
p_{n-1} p_{n}^{2} p_{n+2} \\
2 q_{n-1} q_{n}^{2} q_{n+2}
\end{array}\right\} \\
& \left\{\begin{array}{c}
p_{n-2} q_{n-2} p_{n+2} q_{n+2} \\
p_{n}^{2} q_{n}^{2}
\end{array}\right\} \quad\left\{\begin{array}{l}
p_{n-2} q_{n} p_{n+1} q_{n+2} \\
p_{n-2} p_{n} q_{n+1} p_{n+2}
\end{array}\right\} \quad\left\{\begin{array}{l}
p_{n}^{2} q_{n+1} q_{n+2} \\
q_{n}^{2} p_{n+1} p_{n+2}
\end{array}\right\}
\end{aligned}
$$

We thus have the following pairs of arithmetic progressions in which four of the five terms are Pythagorean ratios:

$$
\begin{array}{cccc}
\left\{\begin{array}{c}
p_{n}^{2} q_{n}^{2} \\
p_{n-2} q_{n-2} p_{n+2} q_{n+2}
\end{array}\right\} & \left\{\begin{array}{l}
p_{n-1} p_{n}^{2} p_{n+2} \\
2 q_{n-1} q_{n}^{2} q_{n+2}
\end{array}\right\} & \left\{\begin{array}{l}
q_{n-2} p_{n-1} q_{n} p_{n+2} \\
p_{n-2} q_{n-1} p_{n} q_{n+2}
\end{array}\right\} *\left\{\begin{array}{l}
q_{n-2} q_{n-1} p_{n}^{2} \\
p_{n-2} p_{n-1} q_{n}^{2}
\end{array}\right\}, \\
\left\{\begin{array}{c}
p_{n-2} q_{n-2} p_{n+2} q_{n+2} \\
p_{n}^{2} q_{n}^{2}
\end{array}\right\} & \left\{\begin{array}{l}
p_{n-2} p_{n}^{2} p_{n+1} \\
2 q_{n-2} q_{n}^{2} q_{n+1}
\end{array}\right\} & \left\{\begin{array}{l}
p_{n-2} q_{n} p_{n+1} q_{n+2} \\
q_{n-2} p_{n} q_{n+1} p_{n+2}
\end{array}\right\} *\left\{\begin{array}{l}
p_{n+1}^{2} q_{n+1} q_{n+2} \\
q_{n}^{2} p_{n+1} p_{n+2}
\end{array}\right\} .
\end{array}
$$

In view of the recurrence relations for the $p_{n}, q_{n}$, these expressions are not unique, but they are the simplest we have found. Among alternative expressions for the other quantities involved, we note that

$$
a_{n}=4 \prod_{i=-2}^{2} p_{n+i} q_{n+i}
$$

which may be halved when $n$ is a multiple of 3 .

\section{REFERENCES}

1. J. Leech, Five tables relating to rational cuboids, Maths. Comp. 32 (1978) 657-659.

2. J. Leech, Two Diophantine birds with one stone, Bull. London Math. Soc. 13 (1981) $561-563$. 
3. J. Leech, Pythagorean ratios in arithmetic progression, Part I. Three, Pythagorean ratios, Glasgow Math J. 35 (1993), 395-407.

Jean Lagrange:

11 RUE Bertrand de Mun

51100 REIMS

France 